\title{
Convergence Properties of a Sequential Regression Multiple Imputation Algorithm: Supplementary Material
}

\author{
Jian Zhu ${ }^{1}$, Trivellore E. Raghunathan ${ }^{2}$
}

Department of Biostatistics, School of Public Health, University of Michigan, Ann Arbor

\section{Proof of Theorem 3}

Proof: As in Theorem 1, we first prove the theorem for exactly specified model sequences, where $m_{i}\left(y_{i} \mid y_{[-i]}, \theta_{i}=\psi_{i}\right)=f_{i}\left(y_{i} \mid y_{[-i]}, \psi_{i}\right)$ for $i=1, \ldots, p$. We need to show that for each regression model, the asymptotic score equation $\tilde{s}_{i}\left(\psi_{i} \mid \psi_{[-i]}\right)$ holds.

Denoting $u_{i}\left(y_{i} \mid y_{[-i]}\right)=\partial \log \left(m_{i}\left(y_{i} \mid y_{[-i]}, \theta_{i}\right)\right) / \partial \theta_{i}$ and $n_{i}$ the sample size of each missingness group, then the asymptotic function for the $i$ th model given $\theta_{[-i]}^{*}=\psi_{[-i]}$ is as

$$
\tilde{s}_{i}\left(\theta_{i} \mid \psi_{[-i]}\right)=\tilde{s}_{i}\left(\theta_{i} \mid R=0\right)+\sum_{j \neq i} \tilde{s}_{i}\left(\theta_{i} \mid \psi_{j}, R=j\right)
$$

where

$$
\tilde{s}_{i}\left(\theta_{i} \mid R=0\right)=\int \cdots \int u_{i}\left(y_{i} \mid y_{[-i]}\right) n_{0} f\left(y_{1}, \ldots, y_{p} \mid R=0\right) \mathrm{d} y_{1} \cdots \mathrm{d} y_{p},
$$

and for $j \neq i$,

$$
\begin{aligned}
\tilde{s}_{i}\left(\theta_{i} \mid \psi_{j}, R=j\right) & =\int \cdots \int u_{i}\left(y_{i} \mid y_{[-i]}\right) n_{j} m_{j}\left(y_{j} \mid y_{[-j]}, \psi_{j}\right) f\left(y_{[-j]} \mid R=j\right) \mathrm{d} y_{1} \cdots \mathrm{d} y_{p} \\
& =\int \cdots \int u_{i}\left(y_{i} \mid y_{[-i]}\right) n_{j} f\left(y_{1}, \ldots, y_{p} \mid R=j\right) \mathrm{d} y_{1} \cdots \mathrm{d} y_{p} .
\end{aligned}
$$

Since the missingness is ignorable, we have

$$
\sum_{j=0, j \neq i}^{p} n_{j} f\left(y_{1}, \ldots, y_{p} \mid R=j\right)=\left(n-n_{i}\right) f\left(y_{1}, \ldots, y_{p} \mid R \neq i\right)=\left(n-n_{i}\right) m_{i}\left(y_{i} \mid y_{[-i]}, \psi_{i}\right) f\left(y_{[-i]} \mid R \neq i\right),
$$

and the asymptotic function can be rewritten as below:

$$
\tilde{s}_{i}\left(\theta_{i} \mid \psi_{[-i]}\right)=\left(n-n_{i}\right) \int \cdots \int u_{i}\left(y_{i} \mid y_{[-i]}\right) m_{i}\left(y_{i} \mid y_{[-i]}, \psi_{i}\right) f\left(y_{[-i]} \mid R \neq i\right) \mathrm{d} y_{1} \cdots \mathrm{d} y_{p},
$$

and it is easy to show that the asymptotic score equation holds:

$$
\tilde{s}_{i}\left(\psi_{i} \mid \psi_{[-i]}\right)=\left.\left(n-n_{i}\right) \int \cdots \int u_{i}\left(y_{i} \mid y_{[-i]}\right) m_{i}\left(y_{i} \mid y_{[-i]}, \psi_{i}\right) f\left(y_{[-i]} \mid R \neq i\right) \mathrm{d} y_{1} \cdots \mathrm{d} y_{p}\right|_{\theta_{i}=\psi_{i}}=0 .
$$

\footnotetext{
1jianzhu@umich.edu

${ }^{2}$ teraghu@umich.edu
} 
Therefore, as $n, t \rightarrow \infty, \theta_{i}^{(t)} \rightarrow \psi_{i}$, which leads to that $m_{i}\left(y_{i} \mid y_{[-i]}, \theta_{i}^{(t)}\right) \rightarrow f_{i}\left(y_{i} \mid y_{[-i]}, \psi_{i}\right)$, for $i=1, \ldots, p$.

We now prove the theorem for validly specified model sequences with extra terms compared to the true conditional densities. As in Theorem 2, suppose that without loss of any generality we introduce a parameterization $\theta_{i}=\left(\zeta_{i}, \xi_{i}\right)$ such that $m_{i}\left(y_{i} \mid y_{[-i]}, \zeta_{i}=\psi_{i}, \xi_{i}=0\right)=f_{i}\left(y_{i} \mid y_{[-i]}, \psi_{i}\right)$. We need to show that $\left\{\theta_{i}^{*}=\left(\psi_{i}, 0\right), i=1, \ldots, p\right\}$ is the convergent point of the asymptotic iterative algorithm.

Given that $\theta_{j}^{*}=\left(\psi_{j}, 0\right)$ for $j \neq i$,

$$
\tilde{s}_{i}\left(\theta_{i} \mid \theta_{[-i]}^{*}\right)=\left(n-n_{i}\right) \int \cdots \int u_{i}\left(y_{i} \mid y_{[-i]}\right) f_{i}\left(y_{i} \mid y_{[-i]}, \psi_{i}\right) f\left(y_{[-i]} \mid R \neq i\right) \mathrm{d} y_{1} \cdots \mathrm{d} y_{p} .
$$

As in Theorem 2, to find the solution to $\tilde{s}_{i}\left(\theta_{i} \mid \theta_{[-i]}^{*}\right)=0$ is equivalent to minimize $\int \cdots \int \log \left[f_{i}\left(y_{i} \mid\right.\right.$ $\left.\left.y_{[-i]}, \psi_{i}\right) / m_{i}\left(y_{i} \mid y_{[-i]}, \theta_{i}\right)\right] f_{i}\left(y_{i} \mid y_{[-i]}, \psi_{i}\right) f\left(y_{[-i]} \mid R \neq i\right) \mathrm{d} y_{1} \cdots \mathrm{d} y_{p}$. Since the relative entropy has non-negative values and its minimum 0 is reached if and only if $m_{i}\left(y_{i} \mid y_{[-i]}, \theta_{i}=\left(\psi_{i}, 0\right)\right)=f_{i}\left(y_{i} \mid\right.$ $\left.y_{[-i]}, \psi_{i}\right)$. Therefore, the asymptotic score equation $\tilde{s}_{i}\left(\theta_{i} \mid \theta_{[-i]}\right)=0$ holds at $\left(\theta_{1}^{*}, \ldots, \theta_{p}^{*}\right)$ for any $i=1, \ldots, p$.

\section{Proof of Theorem 4}

Proof: As in Theorem 2, we first apply the joint model $p\left(y_{1}, \ldots, y_{p} \mid \theta\right)$ to analyze the incomplete data to determine the convergent point. Denote $\tilde{Q}\left(\theta \mid \theta^{(t-1)}\right)$ the expected log-likelihood from the Expectation-Maximization algorithm, where $\theta=\left(\theta_{1}, \ldots, \theta_{p}\right) \in \Theta_{C}$. For $i=1, \ldots, p$, denote $\theta_{M,[-i]}$ the parameter of the marginal joint model of $Y_{[-i]}$ from the joint model, then because of the separability of marginal parameters, $\theta_{M,[-i]}$ is distinctive from $\theta_{i}$ and $\theta=\left(\theta_{i}, \theta_{M,[-i]}\right)$ is a parameterization of the joint model. Since $\theta^{*}=\left(\theta_{1}^{*}, \ldots, \theta_{p}^{*}\right)$ is the maximum likelihood estimate, $\partial \tilde{Q}\left(\theta \mid \theta^{*}\right) /\left.\partial \theta\right|_{\theta=\theta^{*}}=0$. On the other hand, because marginal separability ensures that

$$
\partial \log p\left(y_{1}, \ldots, y_{p} \mid \theta_{i}, \theta_{M,[-i]}\right) / \partial \theta_{i}=\partial\left(\log \left(m_{i}\left(y_{i} \mid y_{[-i]}, \theta_{i}\right)\right)\right) / \partial \theta_{i}=u_{i}\left(y_{i} \mid y_{[-i]}\right),
$$

we have

$$
\begin{aligned}
\partial \tilde{Q}\left(\theta_{i}, \theta_{M,[-i]}^{*} \mid \theta^{*}\right) / \partial \theta_{i} & =\int \cdots \int u_{i}\left(y_{i} \mid y_{[-i]}\right) n_{0} f\left(y_{1}, \ldots, y_{p} \mid R=0\right) \mathrm{d} y_{1} \cdots \mathrm{d} y_{p} \\
& +\int \cdots \int u_{i}\left(y_{i} \mid y_{[-i]}\right) \sum_{j=1}^{p}\left[n_{j} m_{j}\left(y_{j} \mid y_{[-j]}, \theta_{j}^{*}\right) f\left(y_{[-j]} \mid R=j\right)\right] \mathrm{d} y_{1} \cdots \mathrm{d} y_{p} \\
& =\tilde{s}_{i}\left(\theta_{i} \mid \theta_{[-i]}^{*}\right) \\
& +n_{i} \int \cdots \int u_{i}\left(y_{i} \mid y_{[-i]}\right) m_{i}\left(y_{i} \mid y_{[-i]}, \theta_{i}^{*}\right) f\left(y_{[-i]} \mid R=i\right) \mathrm{d} y_{1} \cdots \mathrm{d} y_{p} .
\end{aligned}
$$


Therefore, the asymptotic score equations hold at $\theta^{*}$ as for all $i=1, \ldots, p$,

$$
\begin{aligned}
& \tilde{s}_{i}\left(\theta_{i}^{*} \mid \theta_{[-i]}^{*}\right) \\
= & {\left.\left[\partial \tilde{Q}\left(\theta_{i}, \theta_{M,[-i]}^{*} \mid \theta^{*}\right) / \partial \theta_{i}-n_{i} \int \cdots \int u_{i}\left(y_{i} \mid y_{[-i]}\right) m_{i}\left(y_{i} \mid y_{[-i]}, \theta_{i}^{*}\right) f\left(y_{[-i]} \mid R=i\right) \mathrm{d} y_{1} \cdots \mathrm{d} y_{p}\right]\right|_{\theta_{i}^{*}} } \\
= & 0 .
\end{aligned}
$$

Therefore, as $n, t \rightarrow \infty, \theta_{i}^{(t)} \rightarrow \theta_{i}^{*}$, which leads to that $m_{i}\left(y_{i} \mid y_{[-i]}, \theta_{i}^{(t)}\right) \rightarrow p_{i}\left(y_{i} \mid y_{[-i]}, \theta_{i}^{*}\right)$, for $i=1, \ldots, p$. 